\title{
The effects of interpolated rest on the first and second responses of the conditioned GSR
}

\section{ROYER F. COOK, ${ }^{2}$ Kansas State University, Manhattan, Kans. 66502}

Employing an 8-sec CS-UCS interval, the effect of a rest interval on the first (1.3 to $5.0 \mathrm{sec}$ after $C S$ onset) and second (5.0 to $9.5 \mathrm{sec}$ after CS onset) responses of the GSR was measured. Two groups of 14 Ss received a series of paired tones (CS) and shocks (UCS). The rest interval effected an increase in first responses and a decrease in second responses. The data were taken as further support for the position that first responses contain a large orienting component, while second responses are produced through increments in associative strength.

There have been numerous recent attempts to separate the conditioned GSR from the nonassociative components. Beginning with the work of Stewart and his associates (Stewart, Stern, Winokur, \& Fredman, 1961), the long ( $6-8 \mathrm{sec})$ CS-UCS interval has been employed for these purposes. At present, it appears (Prokasy \& Ebel, 1967) that nonassociative variables affect the responses occurring early in the interval (orienting responses) and associa tive variables affect primarily the responses occurring later in the interval.

It has been shown that the GSR is characterized by a sharp decrease upon frequent presentation of a formerly novel stimulus and a similarly rapid recovery with rest (Sokolov, 1963; Lynn, 1966). The orienting component (OR) of the GSR has a particularly rapid habituation-recovery cycle. In contrast, the conditioned GSR is relatively resistant to extinction (Kimble, 1961 ; Prokasy \& Ebel, 1967).

Consequently, if a rest interval is inserted during a series of conditioning trials, one would expect that after resumption of conditioning the magnitude of the early responses would increase and the magnitudes of the later responses would decrease.

\section{SUBJECTS}

Twelve male and 16 female undergraduates at Kansas State University served as Ss and received \$1.00 each. PROCEDURE

Subjects were presented with five adaptation tones, a series of conditioning trials, i.e., paired tone (CS) and shock (UCS), and four extinction trials. The $2000-\mathrm{Hz}$ tones were delivered at an in tensity of $75 \mathrm{~dB}$ (re 0002 dynes $/ \mathrm{cm}^{2}$ ) for
5 for Group B yields the same general results as the initial comparison. Specifically, the means on Trials 3, 4, and 5 (Group A) are very similar to those of Trials 7,8 , and 9 (Group A). The Group B response to Trial 3 is, therefore, greater than any other responses in either group.

A comparison of Group A second responses on Trials 7, 8, and 9 with Group B second responses on Trials 3, 4, and 5 reveals little difference (obviously nonsignificant) between the two groups. There was a total of only eight second responses on Trials 7,8 , and 9 for Group $A$ and six such responses on those trials for Group B.

In contrast, Group A produced 20 second responses on Trials 3,4 , and 5 . Because of highly skewed data in Group B, the Mann-Whitney U-test was used to test the second response differences between the two groups on Trials 3,4 , and 5 . The comparison yielded a significant difference $[\mathrm{U}(14,14)=43]$ between the groups, greater GSR frequencies resulting from the consecutive conditioning procedure (A) than the interval procedure (B). \section{DISCUSSION}

The central hypothesis in this study was that the stimulation interval would result in first responses of greater magnitude and second responses of lesser magnitude than if the interval were not present. This hypothesis has, for the most part, been confirmed. The data certainly indicate that the introduction of the interval decreases the magnitude of second responses.

However, a more accurate interpretation of the first response data was that the interval yielded a sizeable OR with a significant habituation effect across trials. Such rapid habituation has been noted as characteristic of the OR (Razran, 1961; Zimny \& Schwabe,(1965). This habituation effect was significant while the main effect of the interval was not, largely because of the difference in error terms. The error term for the interval effect was the noticeably

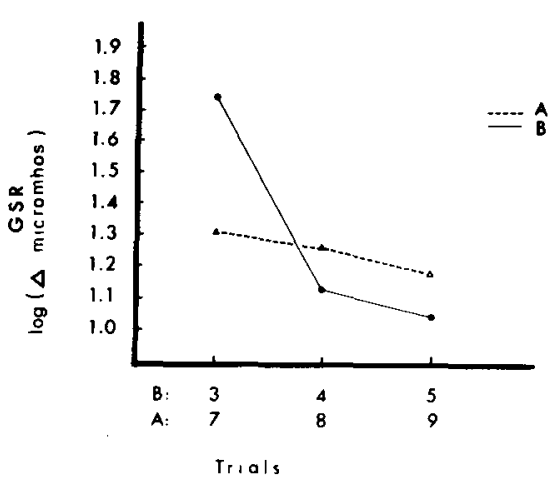

Fig. 1. Mean magnitudes for first responses on Trials 3,4 , and 5 (Group B), and Trials 7, 8, and 9 (Group $A$ ). 
smaller wilhin-S variance. Such lindings suggest that within-Ss designs are eminently applicable to GSR studies.

It is important to note that if these first responses were viewed as $\mathrm{CRs}$, the opposite result would have been expected, i.e., larger first responses would have been expected after a number of conditioning trials than after a stimulation pause.

In contrast to earlier findings by the author (Cook, 1968), there was not a dominant amplitude (discounting zero responses) effect in the larger responses of Group B. In addition to the smaller amplitudes in Group A, there were also four zero responses, as opposed to only one zero response in Group B. This suggests that the larger magnitude obtained in Group B was not only a result of inflated amplitudes, but also reflected increased response probability.

The original hypothesis that Group A second responses on Trials 7,8, and 9 would be larger than Group B responses on Trials 3, 4 , and 5 was not confirmed. The expected effect was found at the earlier Group A trials. The larger second response magnitudes of Group A over Group B appear to be a result of both amplitude and probability, although the probability effect is probably the greater. These findings concur with Prokasy's (1967) data which revealed the second response effect to be almost wholly reflected in response probability.

The second response magnitudes reached a peak in the area from the third to fifth trials. For CRs to peak so quickly seems opposed to conventional reinforcement-repetition effects. However, such findings are not uncommon in the literature (Stewart, Stern, Winokur, \& Fredman, 1961; Prokasy \& Ebel, 1967). Autonomic phenomena, which are usually given the more positive label of conditioning, might more accurately be termed "lack of habituation." Frequently, differences between conditioning groups and pseudoconditioning controls are obtained less from an increase in the conditioning groups than from a decrement in the pseudoconditioning controls.

A conventional explanation for the lack of conditioning in Group B would be that the interval effected a decrement in associative strength or presented the establishment of substantial associative strength in later trials. The implication is that on Trial 3(the initial postinterval trial), $S$ in Group B essentially began anew and lacked the sufficient number of trials to display significant learning in the remainder of the series.

Contrarily, an explanation couched in terms of "perceptual awareness" would contend that the interval had a disruptive effect on S's perception of the stimulus contingencies. Again, there were too few trials for $S$ to perceive the contingencies accurately. In the light of Grings' (1965) work, this interpretation is certainly plausible.

The question concerning the source of the large orienting-first response is an interesting one. The author's recent work indicated that large ORs are caused by stimulus uncertainty. It would seem to follow that the greater $O R$ is a function of a greater uncertainty of tone occurrence in Group B (the interval group). In Group A, S could, to a certain degree, predict the time of the occurrence of the tone and, in some manner, prepare for its reception. In terms of Sokolov's theory (1961), S might be said to be set, or "tuned in," on the proper neuronal model. However, such a process would necessitate a more complex cortical system than the one presently espoused by Sokolov. ${ }^{3}$

A closely related, yet somewhat antithetical, interpretation of the new data would be that an opportunity for OR recovery, rather than uncertainty, is the primary determiner of the greater $O R$. Quite possibly, the habituated OR has sufficient time to recover in the interval.

\section{REFERENCES}

COOK, R. F. Pseudoconditioning of the GSR Unpublished doctoral dissertation, Kansas State University, 1968.
GRINGS. W. W. Verbal-pereptual factors in the conditioning of autonomic responses. In W. $\mathrm{F}$ Prokasy (Ed.), Classical conditioning. New York: Appleton-Century-Crofts, 1964. Pp. $71-89$.

KIMBLE, G. A. Hilgard and Marquis'Conditioning and learning. New York: Appleton-Century-Crofts, 1961.

LYNN, R. Attention, arousal and the orientation reaction New York: Pergamon Press, 1966.

PROKASY, W. F., \& EBEL, A. C. Three components of the classically conditioned GSR in human subject. Joumal of Experimental Psychology, 1967, 73, 247-256.

RAZRAN, G. The observable unconscious and the inferable conscious in current soviet psychophysiology. Psychological Review, 1961, 68, 81-147.

SOKOLOV, E. N. Perception and the conditioned reflex. New York: Pergamon Press, 1963.

STEWART, M. A., STERN, J. A., WINOKUR, G \& FREDMAN, S. An analysis of GSR conditioning. Psychological Review, 1961, 68, 60-67.

TURSKY, B., \& WATSON, P. D. Controlled physical and subjective intensities of electric shock. Psychophysiology, 1964, 1, 151-162.

ZIMNY, G. H., \& SCHWABE, L. W. Stimulus change and habituation of the orienting response. Psychophysiology, 1965, 2, 103-115. NOTES

1. This report is based on the author's doctoral dissertation (Cook, 1968) where additional information may be found. The research was performed while the author was a USPHS Predoctoral Fellow. The author would like to thank Merrill E. Noble for his assistance and guidance through all phases of the research.

2. Now at the American Institutes for Research, Washington Office, 8555 16th Street, Silver Spring, Md. 20910.

3. For a more extensive discussion of such processes, the reader is referred to the author's doctoral dissertation (Cook, 1968).

\section{Visual disappearance of a motion pattern}

\section{E. STURE ERIKSSON, University of Uppsala, Uppsala, Sweden}

An experiment, using static and moving patterns of lines, was performed in order to test the hypothesis that fixation results in a perceptual disappearance of the static pattern, but not in a disappearance of the moving pattern. The results which were obtained verify earlier reported effects with regard to static fading, but also show that fixation yields a total disappearance of the moving pattern of lines. This outcome is interpreted as a complication of the notion of changing stimulation as a necessary and sufficient condition of perception.

In connection with the concept of stimulus within the visual modality, it has long been known that, under the conditions of low illumination, static objects tend to disappear when they are fixated or when the retinal image is stabilized. The effect has been obtained under different conditions. Both foveal and parafoveal regions have resulted in the effect of partial or total fading (Goldstein, 1967, 1968; Kirkwood, 1968).

When discussing Troxler's effect, as well as stabilized retinal images, the major point seems to be the concept of changing stimulation, which implies that changing stimulation is considered as a necessary and sufficient condition for a perceptual effect.

This article offers the establishment of an effect which seems to complicate the notion of changing stimulation, because the result which is reported here consists of a 